\title{
Implementation of Drowsiness Detection and Safe Driving System
}

\author{
SeongSe Cho ${ }^{1}$ and WonHyuk $\mathrm{Choi}^{2}$ \\ ${ }^{1}$ Student, Hanseo University, Korea \\ ${ }^{2}$ Professor, Hanseo University, Korea \\ 1chosse63@gmail.com, ${ }^{2}$ rboooo@naver.com
}

\begin{abstract}
According to recent road traffic accidents statistics, drowsiness driving is about 1.8 times more dangerous than drunken driving, and it's fatal to opponent vehicle driver and pedestrians as well as passengers. In this paper, in order to prevent accidents caused by drowsiness driving, the driver's head attitude data is collected from the sensor mounted on the driver's headset, and the drowsiness and forward gazing state are determined by measuring the angle changes of the head associated with the operation time, We want to implement a system that alerts the drowsy driver according to the result. The drowsiness detection system proposed in this paper was confirmed that it would be easy to manufacture and practical by applying low cost parts.
\end{abstract}

Keywords: Drowsy driving, Drowsy detection, Head angle, Safe driving system

\section{Introduction}

Due to the development of the road and traffic industry, overall system automation/ intelligence for the functions of the vehicle and the safety management of the passengers are being carried out at a rapid pace, but it is still difficult to completely exclude the accident factors caused by human factors.

As shown in [Table 1], According to the analysis of Korea Transportation Industry Research Institute and Road Traffic Corporation, 25,259 accidents caused by drowsiness driving occurred in the last 10 years between 2007 and 2017 in Korea. 1,264 deaths occurred and the mortality rate was $5 \%$. The traffic accident deaths caused by drowsiness driving is more than twice that of the whole traffic accident, and the risk of drowsiness driving is clearly shown with the mortality rate of about 1.8 times higher than the rate of death of drunk driving accidents [1][2].

Table 1. Traffic accidents in recent 10 years (Korea)

\begin{tabular}{|c|c|c|c|c|c|}
\hline \multicolumn{2}{|c|}{ Division } & Period total & Average(year) & Mortality & Remarks \\
\hline \multirow{2}{*}{$\begin{array}{c}\text { Number of } \\
\text { Traffic }\end{array}$} & Total & 2225 thousand & 222.5 thousand & - & \multirow{2}{*}{$2007-2016[1]$} \\
\cline { 2 - 5 } Accidents & Drowsy Driving & 25,259 & $2,525.9$ & - & \\
\cline { 2 - 5 } & Drunk Driving & 255,592 & 25,559 & - & $2008-2017[2]$ \\
\hline \multirow{2}{*}{$\begin{array}{c}\text { Number of } \\
\text { deaths }\end{array}$} & Total & 52,767 & $5,276.7$ & $2.37 \%$ & \multirow{2}{*}{-} \\
\cline { 2 - 5 } & Drowsy driving & 1,264 & 126.4 & $5.00 \%$ & - \\
\cline { 2 - 5 } & Drunk Driving & 7,018 & 701.8 & $2.74 \%$ & - \\
\hline
\end{tabular}

Article history:

Received (November 26, 2019), Review Result (January 14, 2020), Accepted (February 20, 2020) 
In addition, the Korea Transportation Research Institute cited the government traffic statistics in the traffic safety promotion data [3], pointing to the first place among the causes of total traffic accidents as drowsiness driving, the mortality rate is 2.4 times as high as the speeding accident, It is claimed that it is as dangerous as driving in a driver's license cancellation state of $0.17 \%$ of blood alcohol concentration. In Korea, the amendment of the Traffic Safety Law mandates the installation of a Lane Departure Warning System (LDWS) for large-sized business vehicles, four or more axle vehicles, special-purpose vehicles (wing bodies, recharges, moving ladders, etc.) and a policy to support the installation cost subsidy. LDWS is a kind of driver assistant device which is a device that warns the driver when he or she is out of the lane due to drowsiness or carelessness by applying vibration and pressure to the steering device. However, in the case of an ordinary passenger car, it is not mandatory to install it, and in the case of an old vehicle, the user has to install it separately, so in turn, the user does not actively apply it because the installation cost is incurred. In this paper, we have developed a safe driving system that detects the drowsiness pattern according to the driver 's head motion separately from the system installed in the car and provides the result of drowsiness detection according to the head angle change as voice and vibration warning.

\section{Drowsiness driving detection technology research}

Vehicle driver surveillance technology to prevent drowsiness is directly related to the advancement of the automobile industry, leading by the United States, Germany and Japan [4].

Table 2. Domestic and overseas camera-based driver condition monitoring system

\begin{tabular}{|c|c|l|}
\hline Driver's monitoring system & $\begin{array}{c}\text { Development } \\
\text { company }\end{array}$ & Principle of operations \\
\hline $\begin{array}{c}\text { Sleepy Driving Warning } \\
\text { HYUNDAI } \\
\text { MOVIS }\end{array}$ & $\begin{array}{l}\text { Infrared camera mounted inside the vehicle detects driver's } \\
\text { condition through driver's eye movements and facial muscle } \\
\text { changes } \\
\text { Sweat / breathing detection [5], pupil / heart rate [6][7], eye / } \\
\text { nose / mouth detection [8] }\end{array}$ \\
\hline Passenger Eye & $\begin{array}{l}\text { Japan } \\
\text { (Denso) } \\
\text { dashboard recognizes the degree of eyelid change, facial nod, } \\
\text { and lane departure of the vehicle, recognizing it as drowsy } \\
\text { driving and providing warning to the driver. }\end{array}$ \\
\hline 3D Face Tracker & $\begin{array}{c}\text { Japan } \\
\text { (Toyota) }\end{array}$ & $\begin{array}{l}\text { The camera mounted on the front of the instrument panel } \\
\text { recognizes the driver's condition by recognizing the three- } \\
\text { dimensional model change of the driver's face and the face } \\
\text { shape [9]. }\end{array}$ \\
\hline $\begin{array}{c}\text { Attention Assist } \\
\text { Driver Attention } \\
\text { Monitoring System }\end{array}$ & $\begin{array}{c}\text { US } \\
\text { (seeing } \\
\text { machine) }\end{array}$ & $\begin{array}{l}\text { Prediction and warning of driving fatigue due to driving time } \\
\text { and steering level during driving }\end{array}$ \\
\hline $\begin{array}{c}\text { Drowsy driving detection } \\
\text { and alarm system }\end{array}$ & $\begin{array}{l}\text { The camera attached to the steering wheel of the car analyzes } \\
\text { the eye pattern of the driver's eyes and recognizes fatigue and } \\
\text { attention distraction. }\end{array}$ \\
\hline
\end{tabular}

The technology that is mainly applied is image processing utilizing the camera, and the surveillance camera/sensor that is installed in the front part of the driver section to monitor unnecessary actions that distract attention such as sleepiness during driving, and warns the driver. Using the image processing technique, the driver's sensor recognizes the facial area, 
measures changes in the eyelid or the pupil position, etc., and compares it with the database, thereby evaluating the normal operation and determining the corrective action. At this time, the factor considered is the abnormal behavior pattern and the time associated with this behavior. If the abnormal behavior is maintained for a certain period of time, it is determined that the drowsy driving or the concentration is decreased, and a warning is given by voice, sound, vibration and the like. [Table 2] shows the driver monitoring systems based on commercialized image processing for domestic and overseas automobile production companies [4].

In this paper, the sensor used to measure driver's head motion is GY-85. The sensor has an internal accelerometer (ADXL345) [10] that measures the acceleration of gravity in three axes (x, y, z), a gyro (ITG3200) [11] that measures angular velocity in three axes, and a geomagnetic system [12] that measures magnetic fields in three axes (HMC5883L) [Figure 1].
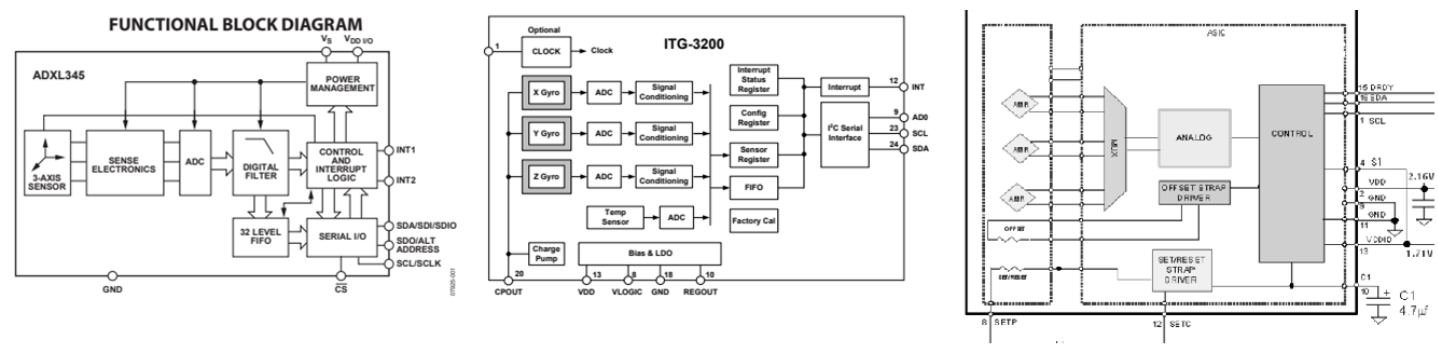

Figure 1. Block Diagram by GY-85 Sensor Module

The accelerometer (ADXL345) is capable of measuring gravity accelerations in the $\mathrm{x}, \mathrm{y}$, and $\mathrm{z}$ axes in a range of \pm 2 to $16 \mathrm{G}$ with 210 resolutions per $\mathrm{g}$. The $\mathrm{z}$-axis is not used because the gravitational vector is invariant, and only the $\mathrm{x}$ - and $\mathrm{y}$-axes are used to calculate the angle [10]. However, the acceleration sensor has a drawback in that it is weak against a small impact and an external noise signal due to its characteristics. Equation (1) (2) (3) is used to calculate the attitude angle using the acceleration sensor. Ideally, when the sensor is perfectly horizontal, there is only gravitational acceleration.

$$
\begin{aligned}
Z_{a c c}=g \times \cos \theta \times \cos \phi \\
Y_{a c c}=g \times \cos \theta \times \sin \phi \\
X_{a c c}=-g \times \sin \phi
\end{aligned}
$$

At this time, if the angle is rotated about the $\mathrm{Y}$-axis by $\Theta$ and the angle about the $\mathrm{X}$-axis is rotated by $\Phi$, the rotation angles of the $\mathrm{X}$-axis and the $\mathrm{Y}$-axis can be obtained from the equations (4) and (5).

$$
\begin{aligned}
& \angle X=\left(\tan ^{-1}\left(\frac{-X_{a c c}}{\sqrt{Y_{a c c}{ }^{2}+Z_{a c c}^{2}}}\right)\right) \times\left(\frac{180}{\pi}\right) \\
& \angle Y=\left(\tan ^{-1}\left(\frac{Y_{a c c}}{\sqrt{{X_{a c c}{ }^{2}+Z_{a c c}{ }^{2}}^{2}}}\right)\right) \times\left(\frac{180}{\pi}\right)
\end{aligned}
$$

\section{Driver drowsiness driving detection system}

\subsection{System algorithm design}


It is ideal for the driver to continuously keep 'watching the progress' for safe driving, except for the necessary manipulation activities such as shifting of the vehicle, signal lamp operation, monitoring of the surroundings / rear situation through the mirror, and so on. However, as traveling times become longer, sleepiness due to fatigue, interference with external environments such as passengers, and deterioration of concentration may result in failure to keep track of the direction of travel. Accumulation of these actions and an increase in the time elapsed will have a fatal impact on safe driving. Based on this assumption, the head of the driver (headset position) is set to the origin $\mathrm{O}$, the traveling direction of the car (front of the driver) is set to the $\mathrm{X}$-axis, the left side of the car is set to the $\mathrm{Y}$-axis, and the vertical direction is set to the $\mathrm{Z}$-axis. The measured range of the sensor-generated by vertical and horizontal head movements of the driver is shown in the form of a cone in [Figure 2a].

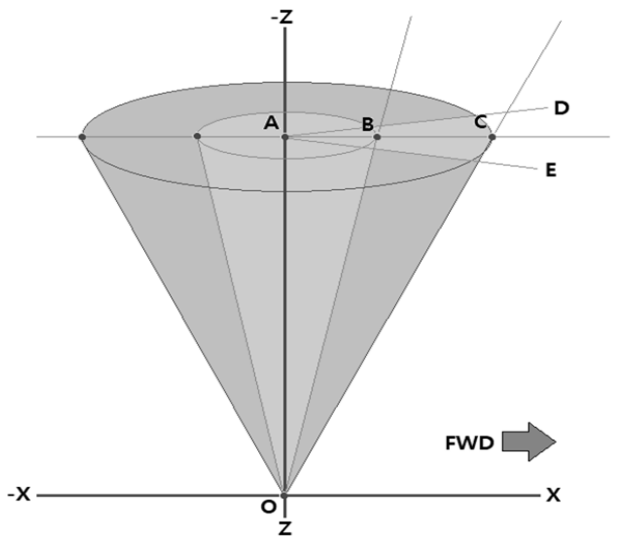

(a)

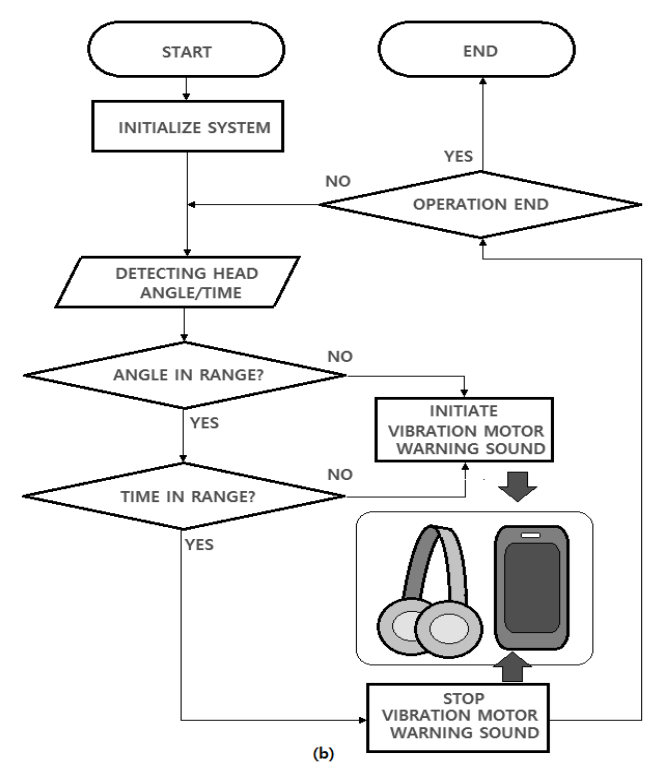

Figure 2. Drowsy driving detection algorithm

The range of the cone including the point $\mathrm{B}$ passing through the $\mathrm{Z}$-axis and the origin $\mathrm{O}$ is a displacement that occurs when performing operations necessary for normal vehicle operation. The range of the cone including the point $\mathrm{C}$ past the $\mathrm{Z}$ axis and the origin $\mathrm{O}$ is the displacement caused by the abnormal movement out of the viewing range of the traveling direction. The angle formed by the straight-line $\mathrm{AD}$ and the straight-line $\mathrm{AE}$ is the same as the range of the displacement in the horizontal direction which occurs while performing the operation necessary for normal vehicle operation. Since the determination range of the normal operation state may vary depending on the behavior characteristics of each driver, it is necessary to set the threshold value through observation and data collection for a predetermined time [Figure 2b]. shows the logical algorithm for detecting drowsy driving or inattentive driving by inputting driver's head movement. When the system is initialized and the head position of the driver equipped with the sensor is set, a continuous signal is inputted every sampling time by the driver's head movement from the sensor based on this. Accordingly, the system converts input data into a changing angle, and determines whether the input data does not deviate from the set limit angle or not exceed the set limit time. 
In the case of a $100 \mathrm{~km} / \mathrm{h}$ speed on the expressway, the distance between the vehicle and the preceding vehicle is maintained at $100 \mathrm{~m}$, which means that it has a braking or avoiding margin of about 3.6 seconds in preparation for stopping the vehicle ahead [3]. Therefore, in this paper, we set the time limit of drowsiness and carelessness to 3 seconds. As a result of judgment, if the deviation is out of the limit angle/time in the vertical direction, a drowsy driving warning is generated. If the limit angle/time in the horizontal direction is exceeded, an inadvertent driving warning is issued. This warning lasts until the condition is cleared. If the condition is cleared, all warning actions will be terminated and the input signal comparison review mode is returned. Drowsy driving or inadvertent driving alerts are provided as voice and video messages through a Bluetooth-enabled smartphone at the same time as vibration and warning sounds through the headset's sensing system. In order to determine the drowsy state by recognizing the driver's head angle change, a filtering step is required to filter the noise due to the error of the sensor itself or the surrounding influence.

\subsection{Drowsy detection system filter design}

We designed a system that required real-time pitch and yaw tilt measurements using the GY-85 with built-in gyro (ITG3200), accelerometer (ADXL345) and magnetometer (HMC5883L). By fusing the measured value of the accelerometer and the measured value of the gyro and appropriately removing the noise, the optimum angle change value can be obtained. In this study, we applied the Extended Kalman Filter (EKF) [13] which shows the most reliable results in the continuously changing external environment and signal processing through the experiment of the previous research.

\subsection{Drowsiness detection system development and verification experiment}

As shown in [Figure 3], the headset including the sensor was manufactured by outputting the self-designed hardware to the 3D printer.

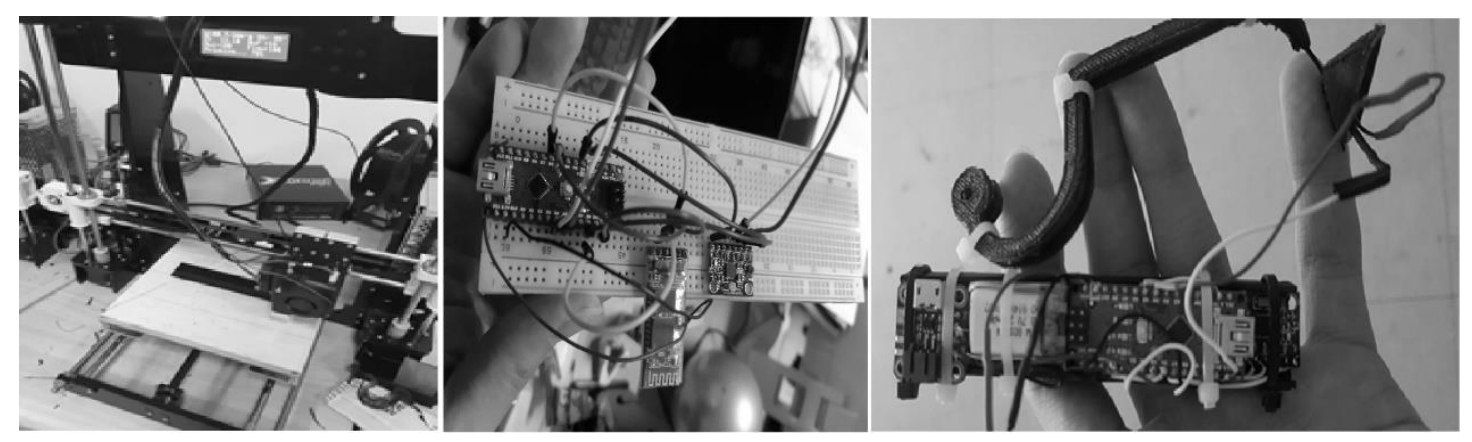

Figure 3. Drowsy detection system hardware

We use this to directly reproduce drowsy operation and careless driving situation in the tired state, and verify the performance and practicality of the designed algorithm and collect the result data. Experimental results show that the performance of the warning system connected with the headset is practical, except for the inconvenience caused by the appearance and the material of the hardware.

[Figure 4] shows the results of about 2 minutes sampling analysis of vertical head movement among the data collected through the headset. The vertical axis represents the angle, and the horizontal axis represents the time $(\mathrm{Sec})$. As predicted from the technical 
analysis of the sensor, the accelerometer continuously generated the over-limit impulse signal due to the external noise and showed a sensitivity to the small vibration.

The gyro initially calculated relatively accurate angle change, but it was observed that drift of about $15^{\circ}$ occurred for 2 minutes due to accumulation of error due to the characteristic of calculating angle change by integrating instantaneous angular velocity. In order to solve this problem, it is confirmed that the signal stability for acquiring the warning condition is secured by applying the extended Kalman filter by fusing the two signals.
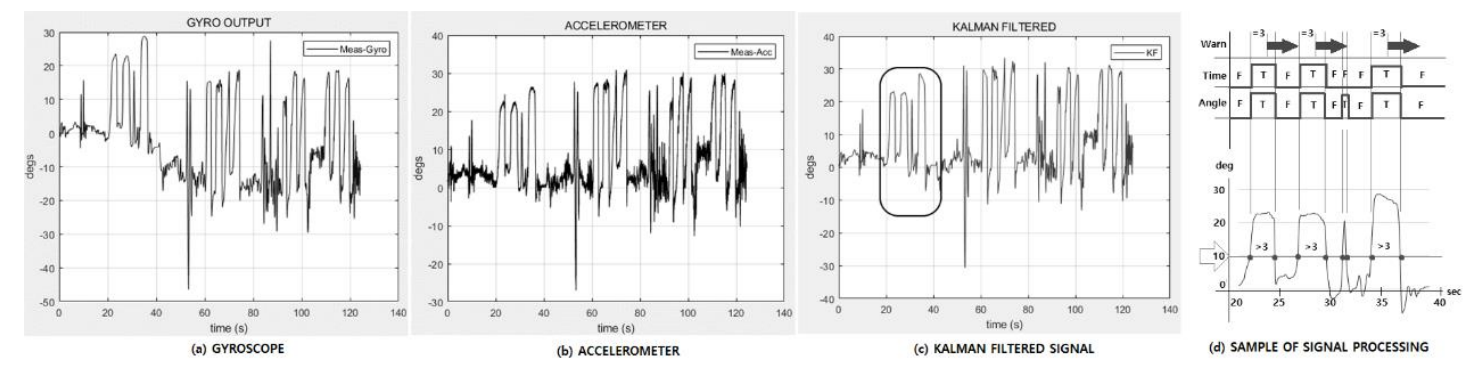

Figure 4. Sensor test results

[Figure. 4(d)] shows an example of the warning signal processing by the processing conditions from the filtered sensor output signal. The example of the head angle change signal is an output signal for 20 seconds, and four times of the state exceeding the limit angle of 10 degrees occurred at this time. There are three cases where the limit time and the limit angle are exceeded. If the two conditions are satisfied through the program logical comparison, it is designed to initialize the warning signal and send commands to the headset and the smartphone. The system worked to the logical criteria we designed.

\section{Conclusion and future research}

In this paper, we propose a method to detect the drowsiness state by recognizing the driver's head inclination angle by attaching sensors to the driver's head independently of the safety driving assist system gradually installed in the new car. In addition, we implemented an alarm system that informs the driver of the dangerous condition according to the detection result. Also, for the convenience of users, it is possible to connect wirelessly through Bluetooth. In order to distinguish it from other systems that have already been commercialized, we have implemented a more effective drowsiness prevention system in cooperation with a smartphone application using a Bluetooth wireless communication as well as a vibration alert of a simple headset. The results of the driver 's wear test that reproduces drowsy driving and careless driving conditions show that the drowsiness detection system proposed in this paper has sufficient manufacturing and practicality by applying appropriate algorithms and filtering using low - cost, off-the-shelf parts.

Future studies will be carried out to further improve the hardware, reduce the size, and reduce the weight of the hardware for the convenience of the driver wearing and using for a long time.

\section{References}

[1] Park, Jeong-wook, "Causes and Preventive Measures of Sleep Driving Traffic Accidents," Bus transportation, vol.55, pp.14-15, (2017)

[2] Park Ji-young, “2008-2017 Drunk Driving Accident Statistical Analysis,” Press Release, (2018) 
[3] "Federation of national bus transport business associations Korea transportation institute," Time Bomb Drowsiness Driving on the Road, (2018)

[4] Choi Kyoung-Im and Kim Joo-young, "Status of Technology Development of Driver's Drowsiness and Inattentive Driving Devices," Monthly Transportation, vol.248, (2018)

[5] H. J. Kim and W. Y. Kim, "Eye detection in facial images using Zernike moments with SVM," ETRI Journal, vol.30, pp.335-337, (2008) DOI:10.4218/etrij.08.0207.0150

[6] Z. H. Zhou and X. Gen, "Projection functions for eye detection," pattern recognition, vol.37, no.5, pp.10491056, (2004) DOI: 10.1016/j.patcog.2003.09.006

[7] J. Qiang and Y. Xiaojie, "Real-time eye, gaze, and face pose tracking for monitoring driver vigilance," Realtime Imaging, vol.8, no.5, pp.357-377, (2002) DOI: 10.1006/rtim.2002.0279

[8] D. F. Dinges and R. Grace, "PERCLOS: A valid psychophysiological measure of alertness as assessed by psychomotor vigilance," Federal Highway Administration, Office of Motor Carriers, (1998) DOI: 10.1037/e509282006-001

[9] T. E. Hutchinson, "Eye movement detection with improved calibration and speed," United States Patent, 4,950,069, (1998)

[10] Wikipedia Contributor, (July 10, 2019), Accelerometer. From Wikipedia, the free encyclopedia. Searched July 22, 2019 at 06:41, https://en.wikipedia.org/w/index.php?title=Accelerometer\&oldid=905654073, (2019)

[11] Wikipedia contributors, (2019, October 24), Gyroscope, In Wikipedia, The Free Encyclopedia. Retrieved 04:13, December 27, 2019, from https://en.wikipedia.org/w/index.php?title=Gyroscope\&oldid=922789200, (2019)

[12] Wikipedia contributors, (2019, July 16), Magnetometer, In Wikipedia, The Free Encyclopedia. Retrieved 06:46, July 22, 2019, from https://en.wikipedia.org/w/index.php?title=Magnetometer\&oldid $=906543745$, (2019)

[13] https://ecse.monash.edu/centres/irrc/LKPubs/Kalman.pdf, Lindsay Kleeman, Department of Electrical and Computer Systems Engineering Monash University, Clayton, Understanding and Applying Kalman Filtering, (2008)

\section{Authors}

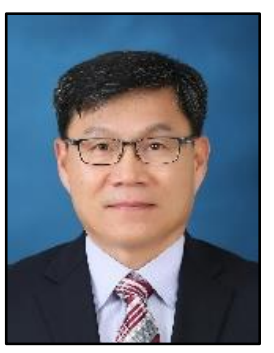

\section{Seongse Cho}

Received his bachelor's degree in computer science from the Korea National Open University in 2003. In 2017, he received the Master degree in avionics engineering from Hanseo University, Seosan, Korea. His research interests include military fighter jet avionics system, UAV collision avoidance, robotics, and intelligent transportation systems.

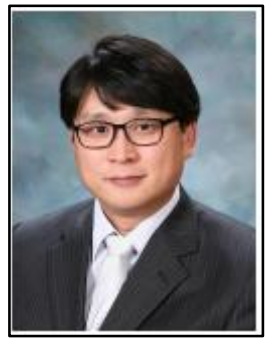

\section{Wonhyeok Choi}

Received his Doctor of Philosophy degree from the Korea Aerospace University, in 2006. During 2008-2014, he has been a professor at Doowon University. He is currently a professor of Avionics Engineering, Graduate School of Hanseo University. He has participated in various projects related to embedded systems and the internet of things, hosted by the state and enterprises. His research interests include embedded systems, IoT, Wireless communication, home-networks \& automation, robot/UAV control. 
Implementation of Drowsiness Detection and Safe Driving System

This page is empty by intention. 